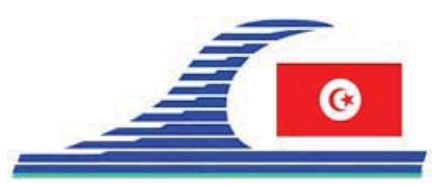

Conférence Méditerranéenne Côtière et Maritime EDITION 1, HAMMAMET, TUNISIE (2009)

Coastal and Maritime Mediterranean Conference

Disponible en ligne - http://www.paralia.fr-Available online

\title{
Morphologie et sédimentologie d'un environnement littoral abrité : cas de la lagune d'Aouled Ridha, Sud-Est tunisien
}

\author{
Faten TELAHIGUE ${ }^{1}$, Younes JEDOUI ${ }^{1}$, Mabrouk MONTACER ${ }^{2}$
}

1. Unité de Recherche « Hydrosciences Appliquées » (06/UR/10-03),

Université de Gabès, Institut des Sciences et Techniques des Eaux de Gabès, Zerig, 6072 Gabès, Tunisie.

Faten_telahigue@yahoo.fr ; Younes.jedoui@issteg.rnu.tn

2. Unité de Recherche " GEOGLOB » (03/UR/10-02), Université de Sfax,

Faculté des Sciences de Sfax, route de Soukra, 3018, Sfax, Tunisie.

isstegb@isstegb.rnu.tn

\section{Résumé :}

La lagune d'Aouled Ridha, située à $56 \mathrm{~km}$ au sud de la ville de Sfax, constitue un bon exemple d'une côte basse, abritée et relativement confinée. Elle présente trois éléments morphologiques typiques des milieux lagunaires côtiers, à savoir: (1) une zone intertidale relativement vaste, subdivisée en slikke et schorre séparés par une microfalaise, (2) une zone supratidale, (3) des chenaux de marée de différentes tailles. Les figures sédimentaires discernables à marée basse dans la zone intertidale telles que les rides, les traces d'activité animale et les tapis cyanobactériens, reflètent la faiblesse de l'énergie hydrodynamique du milieu de sédimentation. L'étude de telles structures sédimentaires, généralement bien préservées, dans les séries anciennes est un outil de détermination des conditions de milieu qui reignaient au cours du dépôt du sédiment. Les sédiments superficiels de la lagune sont formés essentiellement par des sables vaseux fins à très fins, modérément à modérément bien classés. Les sédiments lagunaires sont constitués de quartz, de feldspath, de calcite, de dolomite, et d'une fraction argileuse composée d'attapulgite, illite, kaolinite et de smectite. La matière organique contenue dans le sédiment est d'origine algaire et est immature.

Mots clés : Morphologie - Sédimentologie - Dynamique côtière - Minéralogie Géochimie organique - Lagune d'Aouled Ridha - Côte sud de Sfax

\section{Introduction}

La lagune d'Aouled Ridha est séparée de la mer par un cordon assez bas (voir figure 1). La communication entre la lagune et la mer est assurée par un chenal de marée. Le marnage qui atteint 1,6 m en période des vives eaux, favorise le développement d'une zone intertidale relativement vaste avec une variété de structures sédimentaires qui contribuent à une bonne caractérisation des conditions hydrodynamiques et sédimentologiques de la lagune.

DOI: $10.5150 / \mathrm{cmcm} .2009 .040-5$ 


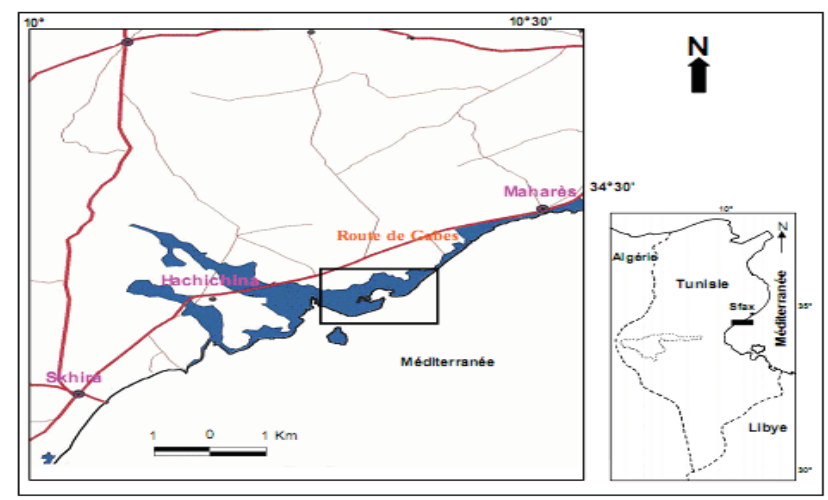

Figure 1. Carte de localisation de la lagune d'Aouled Ridha (extrait de la carte topographique 1/200000, feuille de Sfax ; 1987).

Trente deux échantillons de surface ont été prélevés de la lagune (voir figure 2) et ont fait l'objet d'études sédimentologique (tamisage humide et sec), minéralogique (diffraction des rayons $\mathrm{X}$ ) et organo-géochimique (extraction et fractionnement isotopique, CPG et Spectrométrie de Masse)

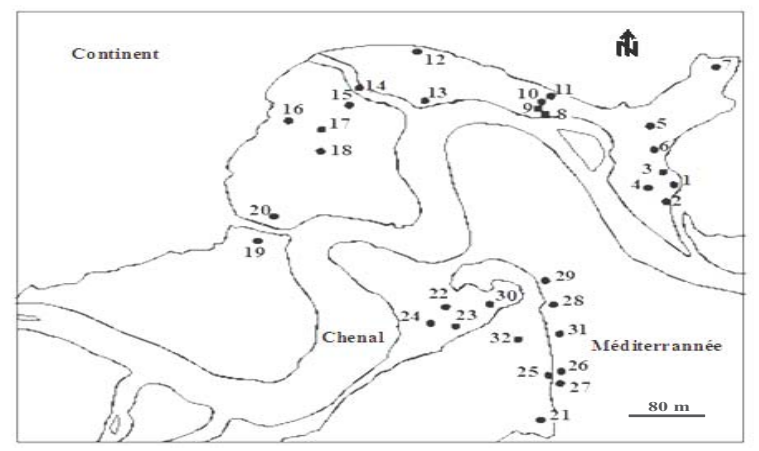

Figure 2. Carte de localisation des échantillons de la lagune d'Aouled Ridha.

\section{Morphologie}

La lagune d'Aouled Ridha, d'une superficie d'environ $3 \mathrm{~km}^{2}$, est un petit bassin abrité, allongé parallèlement à la ligne de rivage. Elle est séparée de la mer par un ancien cordon littoral constitué de sables calcaires, d'âge holocène, recouverts de limons rouges continentaux (JEDOUI, 1979). La lagune présente trois unités morphologiques distinctes :

\subsection{La zone intertidale}

C'est la zone de balancement des marées. Elle présente deux parties, à savoir, la slikke et le schorre séparés par une microfalaise très bioturbée. La slikke, couverte en partie par des Cymodocées et d'algues (essentiellement l'Ulva), se trouve inondée à chaque marée haute. Ses sédiments mous et très bioturbés, sont tapissés, par endroits, par des volcans de boue. Le schorre, occupé par une végétation serrée de salicornes, et qui 
développe des fentes de dessiccation et des tapis cyanobactériens, n'est recouvert que par les marées hautes de vives-eaux.

\subsection{Les chenaux de marée}

Ils assurent les échanges entre la lagune et la mer par les courants de flot et de jusant. Dans la lagune, il existe un chenal principal méandriforme qui présente des ramifications correspondant à des chenaux de marée secondaires dont les affluents sont des chenaux tertiaires. Leurs fonds sont tapissés de rides de courant de petite taille plus ou moins sinueuses, ce qui reflète le caractère moyennement puissant des courants de marée dont la vitesse ne dépasse pas $0,15 \mathrm{~m} / \mathrm{s}$ (GUILLAUMONT, 1992).

\subsection{La zone supratidale}

Située au dessus du niveau de la marée haute, cette zone se trouve toujours hors de l'eau. Sa limite inférieure est marquée par l'accumulation de débris de feuilles de Posidonies et de Cymodocées.

\section{Nature et répartition des faciès sédimentaires}

Deux faciès principaux ont été dégagés en fonction du pourcentage relatif de la fraction fine (diamètre $<63 \mu \mathrm{m}$ ) contenue dans le sédiment :

- un faciès vaseux (fraction fine $>30 \%$ ), caractérisant les endroits les plus abrités de la lagune essentiellement derrière le cordon littoral;

- un faciès sablo-vaseux (fraction fine $<30 \%$ ), occupe la plus grande superficie de la lagune. Les sédiments les plus grossiers sont localisés essentiellement sur les berges des chenaux de marée.

Le sédiment est essentiellement fait de sables fins à très fins $(2,64 \phi<\mathrm{Mz}<3,99 \phi)$. Les courbes cumulatives sont dans l'ensemble assez étalées, essentiellement pour les échantillons 4 et 12 (voir figure 3). Les sables sont dans l'ensemble modérément classés à mal classés $(0,71<\sigma<1,57)$. Quelques échantillons présentent des courbes moyennement redressées, ce sont des sables, modérément bien classés $(0,59<\sigma<0,7)$, situés dans les endroits les plus abrités de la lagune.
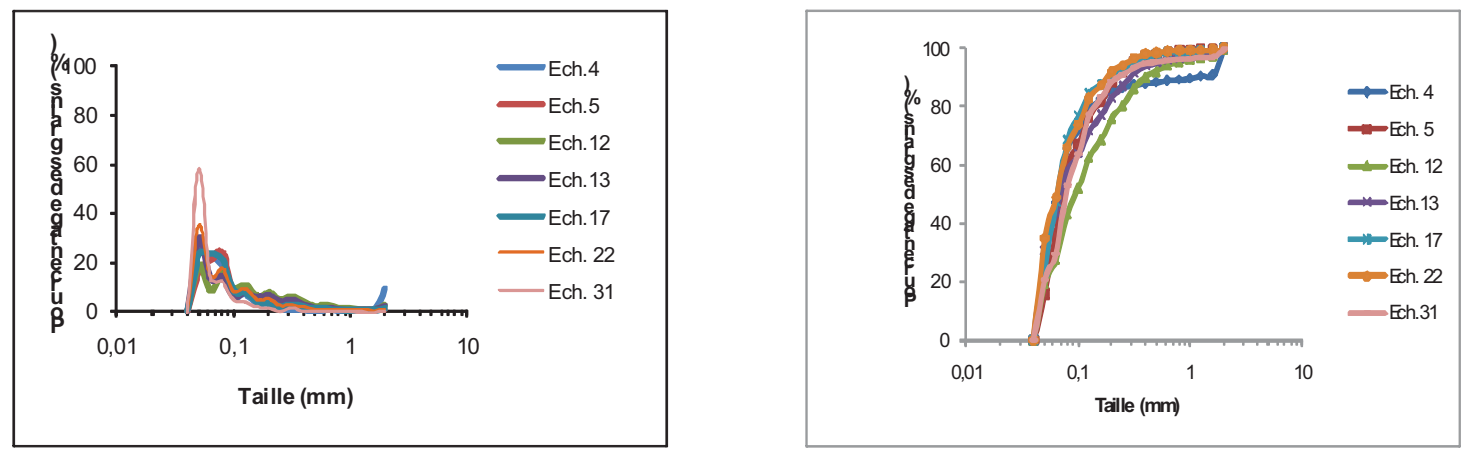

Figure 3. Courbes de fréquence et cumulatives de quelques sédiments de la lagune. 


\section{Composition minéralogique}

La fraction argileuse des sédiments de la lagune est composée d'attapulgite, illite, kaolinite et smectite. La présence de l'attapulgite provient de l'érosion des formations continentales mio-pliocènes bordières. Une présence pareille a été prouvée dans les sédiments continentaux mio-pliocènes de Maknassy et de Gabès (JEDOUI, 1979).

\section{Contenu organique}

La matière organique contenue dans les sédiments, de par ses faibles taux de l'extrait lipidique (de $0,06 \mathrm{mg} \mathrm{HC/g}$ à $0,46 \mathrm{mg} \mathrm{HC/g}$ de roche) et de la prédominance des composés NSO (de 64\% à 97\% de l'extrait total) par rapport aux hydrocarbures saturés et aromatiques, est une matière organique récente et très peu évoluée. La distribution unimodale en $\mathrm{n}-\mathrm{C} 23$, reflète une origine algaire de la matière organique (VENKATESAN et al., 1987). Les teneurs en n-C17 et n-C18 sont nettement supérieures à celles des Pristane et Phytane, ce qui plaide en faveur d'une contamination du site par des hydrocarbures plus matures d'origine allogène, par la déversion du fioul par les barques de pêche abondantes dans la lagune.

\section{Conclusions}

La lagune d'Aouled Ridha est constituée d'une zone intertidale subdivisée en slikke et schorre, d'une zone supratidale et des chenaux de marée. Les sédiments de surface sont constitués essentiellement de sables fins à très fins, sablo-vaseux à vaseux, modérément bien classés Les sédiments superficiels de la lagune contiennent principalement du quartz et accessoirement de la calcite, de la dolomite et des feldspaths. La fraction argileuse assez hétérogène, constituée d'attapulgite, d'illite, de kaolinite et accessoirement de smectite, est le reflet d'un héritage sédimentaire, dû à l'érosion des formations continentales bordières. La matière organique algaire et immature, contenue dans le sédiment, est bien préservée.

\section{Références bibliographiques}

GUILLAUMONT B. (1992). Etude des masses d'eau du golfe de Gabès par Télédetection. Rapport national sur la pollution marine dans le golfe de Gabès et son impact sur l'environnement, Centre National de Télédetection, 23 p.

JEDOUI Y. (1979). Etude hydrologique et sédimentologique d'une lagune en domaine méditerranéen : la Bahiret El Boughrara (Tunisie). Thèse de $3^{\mathrm{ème}}$ cycle, Université de Bordeaux, $190 \mathrm{p}$.

VENKATESAN M.I., RUTH E., STEINBERG S., KAPLAN I.R. (1987). Organic geochemistry of sediments from the continental margin of Southern New England, USA: Part II. Lipids. Marine Chemistry 21, pp 267-299. 